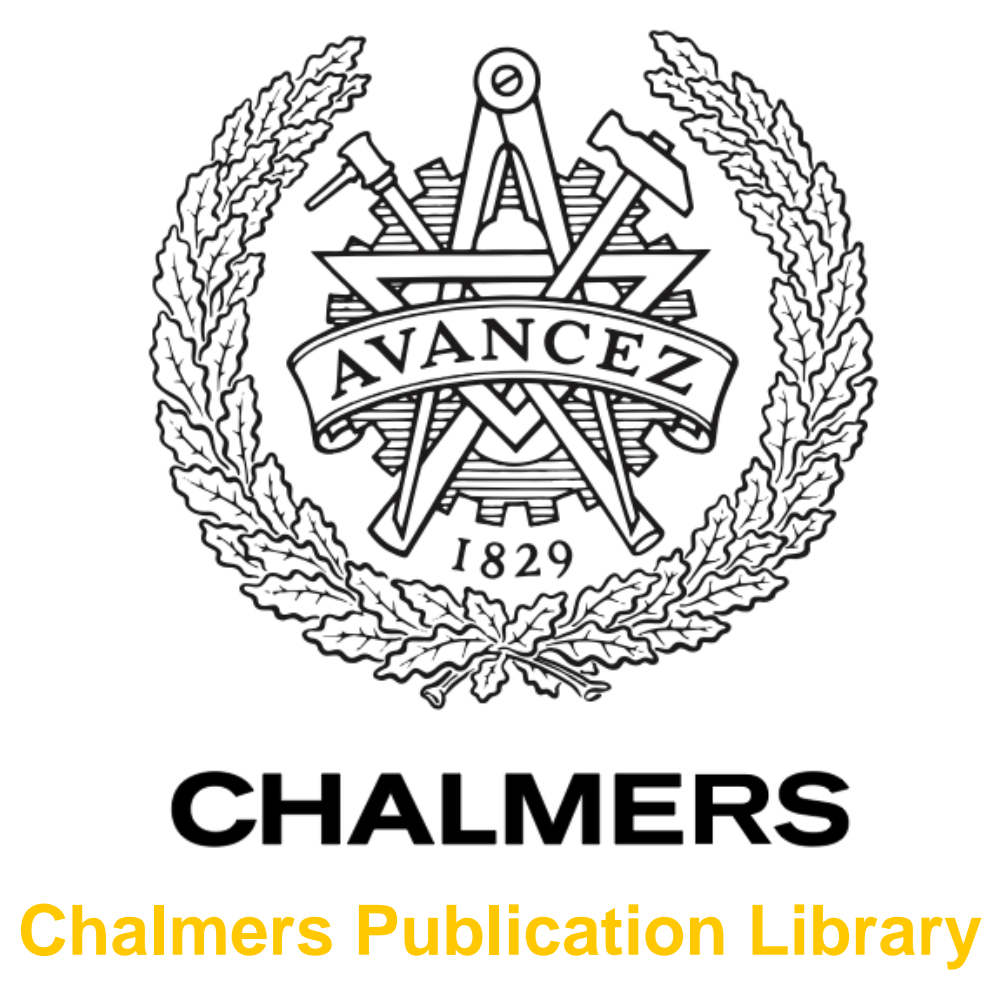

\title{
Comparison between Coherent Superposition in DSP and PSA for Mitigation of Nonlinearities in a Single-span Link
}

This document has been downloaded from Chalmers Publication Library (CPL). It is the author's version of a work that was accepted for publication in:

Optical Communication (ECOC), 2014 European Conference on

\author{
Citation for the published paper: \\ Eliasson, H. ; Olsson, S. ; Karlsson, M. (2014) "Comparison between Coherent Superposition \\ in DSP and PSA for Mitigation of Nonlinearities in a Single-span Link". Optical \\ Communication (ECOC), 2014 European Conference on pp. 3.
}

http://dx.doi.org/10.1109/ECOC.2014.6964015

Downloaded from: http://publications.lib.chalmers.se/publication/206902

Notice: Changes introduced as a result of publishing processes such as copy-editing and formatting may not be reflected in this document. For a definitive version of this work, please refer to the published source. Please note that access to the published version might require a subscription. 


\title{
Comparison between Coherent Superposition in DSP and PSA for Mitigation of Nonlinearities in a Single-span Link
}

\author{
Henrik Eliasson, Samuel L.I. Olsson, Magnus Karlsson, Peter A. Andrekson
}

Photonics Laboratory, Department of Microtechnology and Nanoscience. Chalmers University of Technology, SE-412 96, Gothenburg, Sweden. Email: henrik.eliasson@chalmers.se

\begin{abstract}
An experimental comparison is made between nonlinearity mitigation through coherent superposition optically in a phase-sensitive amplifier and electrically in DSP. The improved nonlinear tolerance is quantified in terms of EVM with high received power and sensitivity with high launch power.
\end{abstract}

\section{Introduction}

Coherent superposition of a signal and its conjugate is a powerful method for mitigating nonlinear distortions. Previously, mitigation of nonlinearities by coherent superposition has been demonstrated both in the optical domain with phase-sensitive amplifiers (PSA) based on four-wave mixing $(\mathrm{FWM})^{1}$, and in the electrical domain using digital signal processing (DSP) with a coherent receiver ${ }^{2,3}$. The conjugate can be transmitted e.g. on a separate wavelength, as is the case with a non-degenerate PSA, or on an orthogonal polarization state, which is the case with phase-conjugated twin waves (PCTW) ${ }^{2}$. Recently PCTW was demonstrated with the conjugate condition fulfilled over the whole signal spectrum of several channels in order to further mitigate inter-channel nonlinearities ${ }^{4}$. In order to maximize the mitigation of nonlinearities it is important to optimize the dispersion map of the link. When using PCTW in a long-haul link without inline dispersion compensation the optimal dispersion map is symmetric $^{2}$. For a single-span this is not the case since the power evolution is strongly anti-symmetric. The optimal dispersion map for single-span transmission has previously been investigated both numerically and experimentally ${ }^{5}$. When using a PSA, the dispersion compensation has to be done in the optical domain while with a DSP-based approach electrical dispersion compensation (EDC) can be used. Using an optimized dispersion map, it can be shown that the nonlinear distortions on the signal and the conjugate are anticorrelated to lowest order ${ }^{2}$. By cancelling nonlinear distortions to lowest order using coherent superposition, the launch power can be increased giving a higher optical signal-to-noise ratio (OSNR). Coherent superposition of a signal symbol $E_{S}$ and a conjugate symbol $E_{C}$ is performed according to $E_{C S}=E_{S}+\left(E_{C}\right)^{*}$, where $E_{\mathrm{CS}}$ is the coherent superposition symbol. In this paper we make a direct comparison between the performance, in terms of nonlinear tolerance, of a system which performs the coherent superposition in the optical domain with a PSA and a system which performs the coherent superposition in DSP. To the best of our knowledge, this is the first time the two approaches have been studied in the same experimental setup. In order to do a fair comparison we use the same transmitter, link and coherent receiver for both systems. The conjugate, sometimes called idler when transmitted on a separate wavelength, is generated using a fiber optic parametric amplifier (FOPA).

\section{Experimental Setup}

Experiments were conducted to investigate the performance of nonlinearity mitigation through coherent superposition in single-span transmission. The schematic of the experimental setup is shown in Fig 1. A $300 \mathrm{kHz}$ linewidth signal laser at $1549.5 \mathrm{~nm}$ was modulated with 10 GBaud QPSK data using an IQ-modulator before feeding the signal together with a CW pump at $1553.5 \mathrm{~nm}$ into a FOPA, the copier, which generated a phase conjugated copy at 1557.5 $\mathrm{nm}$. After the copier the pump was separated from the signal and conjugate using a wavelength division multiplexing (WDM) coupler and attenuated to $5 \mathrm{dBm}$ into the link. The signal and conjugate was passed through an optical processor (OP) in order to balance their optical powers. The generated waves were then dispersion pre-compensated in an optimal manner for 10 GBaud data by the equivalent of 23.6 km SMF ${ }^{5}$ using a section of dispersion compensating fiber (DCF) before being amplified by an EDFA to the selected link launch power and transmitted into a 105 $\mathrm{km}$ fiber span. At the end of the link, the residual dispersion was compensated using a fiber Bragg grating (FBG) dispersion compensating module (DCM). Three different pre-amplifier/receiver setups were compared. In the first, an EDFA was used as pre-amplifier and in this case both the signal and conjugate were separately detected with a coherent receiver and the symbols coherently superposed in offline processing. In the second, the signal was pre-amplified with a phase-insensitive amplifier (PIA) with $15.3 \mathrm{~dB}$ net gain and phase-insensitive operation was achieved by blocking the conjugate after the copier in the OP. The third receiver used a PSA that amplifies and performs the coherent superposition of signal and conjugate all-optically, the PSA had a net gain of $20.8 \mathrm{~dB}$. The gain medium for the PIA and PSA was a cascade of strained highly nonlinear fibers (HNLF) with isolators between each segment to passively suppress stimulated Brillouin scattering (SBS). The cascade was similar to the one used $\mathrm{in}^{6}$. In the case of PIA and PSA, the signal was detected in the $90^{\circ}$ hybrid but not the conjugate. For the PIA and PSA receivers, a pump recovery (PR) stage was used before the parametric amplifier in order to regenerate and amplify the 


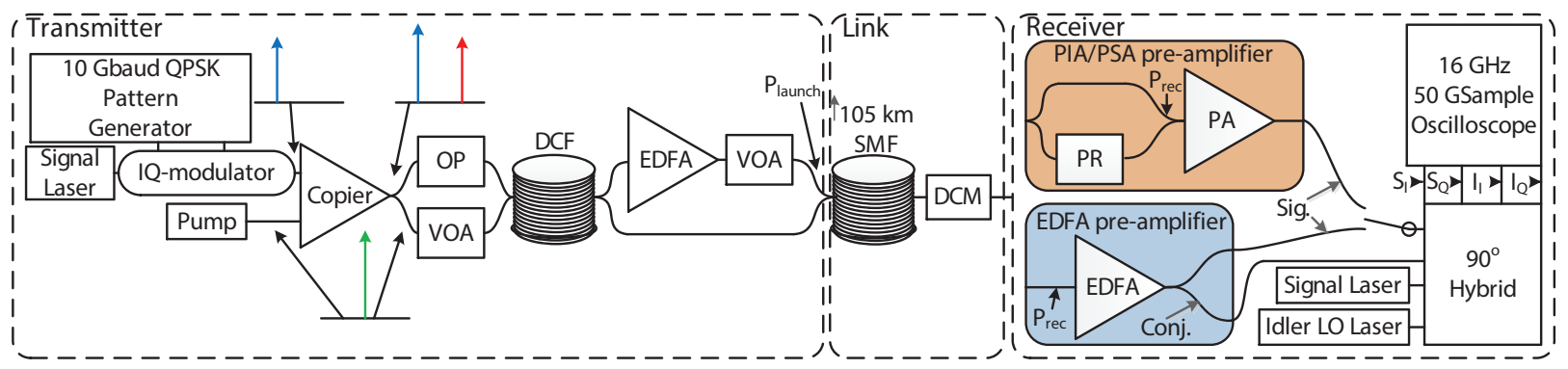

Fig. 1. The experimental setup with transmitter, link and receiver with two different pre-amplifier configurations. Optical processor (OP), variable optical attenuator (VOA), dispersion compensating fiber (DCF), erbium-doped fiber amplifier (EDFA), single-mode fiber (SMF), dispersion compensating module (DCM), pump recovery (PR), phase-insensitive amplifier (PIA), phase-sensitive amplifier (PSA), parametric amplifier (PA)

pump. The details of the pump recovery stage can be found $\mathrm{in}^{7}$. The coherent detection was done with an integrated $90^{\circ}$ hybrid receiver and a $50 \mathrm{GS} / \mathrm{s}$ real-time oscilloscope was used for analog-to-digital conversion. The signal laser was also used as local oscillator for the signal while the conjugate was detected using a free running LO laser, this is not expected to affect the results since the signal was transmitted over a $105 \mathrm{~km}$ link which is much longer than the coherence length of the laser. The offline DSP consisted of downsampling to 2 samples per symbol, 5-tap single-polarization constant modulus algorithm (CMA) equalization and phase tracking using the Viterbi-Viterbi algorithm followed by BER counting on the signal, conjugate and coherent superposition in the EDFA case and the signal in the PIA and PSA case.

\section{Results and Discussion}

To study the performance of the nonlinearity mitigation, error vector magnitude (EVM) measurements were performed with different link launch powers. The link launch power was measured before the link, at $P_{\text {launch }}$ in Fig. 1. To quantify the improvement in the high launch power regime we compare the increase in EVM of the signal and coherent superposition with the EDFA pre-amplifier to the EVM of the signal with PIA and PSA pre-amplifiers. The results from these measurements are shown in Fig. 2(a). In the figure we see that the EVM increases at a lower rate for the systems utilizing coherent superposition, i.e. the EDFA and PSA setups. The EVM increase at high launch powers is lowest for the system performing the coherent superposition in DSP. The specified launch power is the power of the signal not accounting for the conjugate so that the total power of both signal and conjugate copy launched into the fiber is $3 \mathrm{~dB}$ higher for the EDFA and PSA case. We also see that for the systems utilizing coherent superposition (EDFA and PSA), the launch power can be increased to $18 \mathrm{dBm}$ without incurring an $\mathrm{EVM}^{2}$ penalty larger than $1 \mathrm{~dB}$ per $\mathrm{dBm}$ of increased launch power. We use this as a criteria for the launch power at which we perform measurements of the BER as a function of received signal power since $\mathrm{EVM}^{2} \propto \mathrm{SNR}$. In order to see how the systems perform in a regime with both strong nonlinear effects and Gaussian noise due to low received optical power, we performed sensitivity measurements at $18 \mathrm{dBm}$ launch power. The received optical power was measured before the pre-amplifier, see $P_{\text {rec }}$ in Fig. 1. The results from these measurements are shown in Fig. 2(b). We can see that the improvement from coherent superposition is larger at low BER where the nonlinearities are dominating. We also see that the BER for the EDFA signal and conjugate reach a floor caused by nonlinearities at high received powers (>-39 $\mathrm{dBm})$. The penalty of the system doing coherent superposition in DSP compared to the PSA at BER $=10^{-3}$ is $2.7 \mathrm{~dB}$ and at $B E R=10^{-4}$ it is $2.4 \mathrm{~dB}$. This penalty is due to the higher noise figure of the EDFA. That the penalty is lower at $B E R=10^{-4}$ indicates that the DSP system mitigates nonlinearity slightly more efficient since the nonlinearities affect the BER to a larger extent at low BER and high launch power. It should be pointed out that the performance of the PSA could be penalized by the fact that the residual dispersion was not perfectly compensated in the experiment leading to the timing of the signal and idler into the PSA being slightly off. Another thing worth to note is that when using DSP coherent superposition, the DSP is operating on severely nonlinearly distorted signals and e.g. the Viterbi-Viterbi algorithm could perform suboptimally. There are also some differences between the DSP and PSA approach in terms of receiver hardware, dispersion compensation and achievable noise figure. One important difference is that a system performing the coherent superposition in DSP requires two $90^{\circ}$ hybrid receivers since the signal and conjugate copy have to be detected separately. Since a PSA does the coherent superposition in the optical domain, only the signal needs to be detected. Another important area where the systems differ is dispersion compensation. The PSA requires the dispersion to be compensated optically before the signal and idler waves are launched into the PSA. When using a DSP approach, the dispersion can be both pre- and post-compensated using EDC. The last major fundamental difference is the noise figure of the pre-amplifiers. An EDFA preamplifier cannot have a noise figure lower than $3 \mathrm{~dB}$ while a PSA has a $0 \mathrm{~dB}$ quantum limited noise figure ${ }^{8}$. These differences are highlighted in Table. 1. 
Table 1. Table highlighting fundamental differences between systems performing coherent superposition in a PSA and in DSP.

\begin{tabular}{|l||l|l|}
\hline & PSA coherent superposition & EDFA amplified DSP coherent superposition \\
\hline \hline Receiver hardware & $1 \times 90^{\circ}$ hybrid receiver & $2 \times 90^{\circ}$ hybrid receivers \\
\hline Dispersion compensation & Requires optical compensation & Can use EDC \\
\hline Quantum limited noise figure & $0 \mathrm{~dB}$ & $3 \mathrm{~dB}$ \\
\hline Requires phase locked waves & Yes & No \\
\hline
\end{tabular}

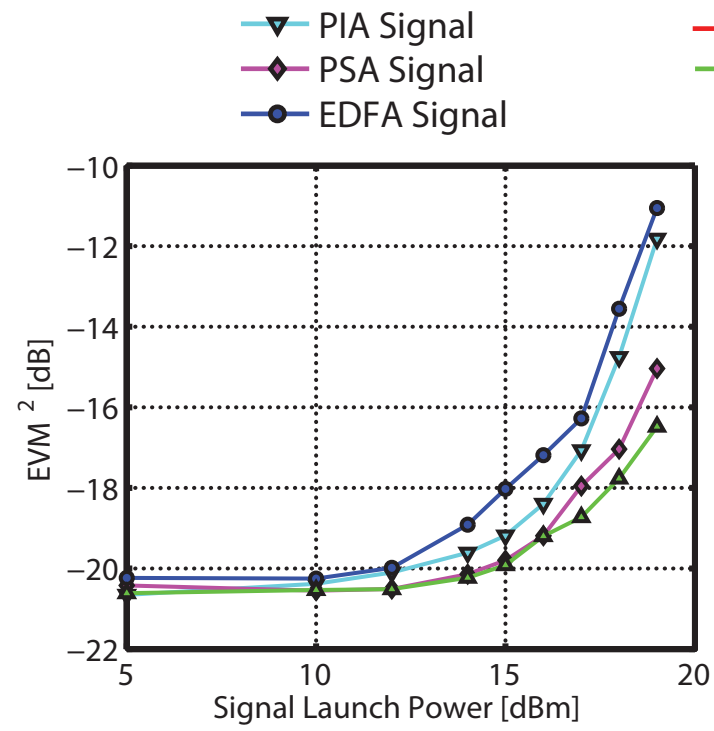

(a)
EDFA Conjugate

EDFA Coherent Superposition

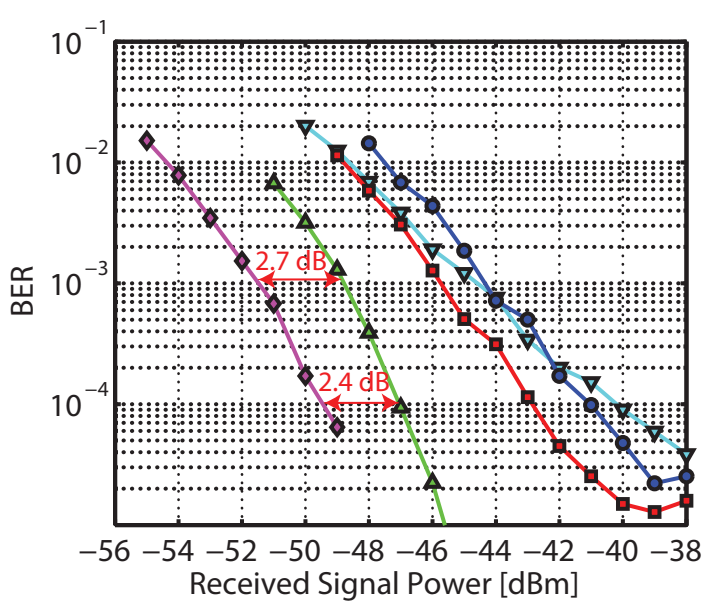

(b)

Fig. 2. (a): $E V M^{2}[\mathrm{~dB}]$ at different signal launch powers. The received signal power is tuned in all cases so that the $E V M^{2}$ is approximately $-20.5 \mathrm{~dB}$ at $5 \mathrm{dBm}$ launch power. (b): BER as a function of received signal power at $18 \mathrm{dBm}$ launch power for the different systems.

\section{Conclusions}

We have made the first direct comparison between mitigation of nonlinearities through coherent superposition electrically in DSP and optically in a PSA. An effort was made to make the two systems as similar as possible in order to focus only on the mitigation of nonlinearities. The focus has been on investigating if there are significant differences between the two approaches in terms of performance in the nonlinear domain. We have measured EVM at different launch powers and performed sensitivity measurements at 18 $\mathrm{dBm}$ signal launch power and compared the penalty at different $B E R$ in order to quantify the performance of the nonlinear mitigation. The PSA and DSP based coherent superposition approaches show very similar performance. When going from BER $=10^{-3}$ to $B E R=$ $10^{-4}$, the penalty of the DSP coherent superposition system compared to the PSA system is decreased from 2.7 to $2.4 \mathrm{~dB}$. This together with the lower rate of increase of EVM at high launch powers indicates that the DSP coherent superposition system cancelled nonlinearities slightly more efficient than the PSA system in our experiment. Finally we have pointed out some important fundamental differences between the two different implementations of coherent superposition.

\section{Acknowledgements}

This work is supported by the European Research Council under grant agreement ERC-2011-AdG 291618 PSOPA, by the Swedish Research Council (VR), and by the Wallenberg Foundation. The authors would like to thank OFS Denmark for providing HNLFs.

\section{References}

[1] S. L. I. Olsson et al., "Phase-Sensitive Amplified Optical Link Operating in the Nonlinear Transmission Regime", Proc. ECOC'12, Th.2.F.1 (2012).

[2] X. Liu et al., "Phase-conjugated twin waves for communication beyond the Kerr nonlinearity limit", Nat. Phot., 7, 560-568 (2013).

[3] Y. Tian et al., "Demonstration of digital phase-sensitive boosting to extend signal reach for long-haul WDM systems using optical phaseconjugated copy", Opt. Exp., 21, 5099-5106 (2013).

[4] X. Liu et al., "Generation of 1.024-Tb/s Nyquist-WDM phaseconjugated twin vector waves by a polarization-insensitive optical parametric amplifier for fiber-nonlinearity-tolerant transmission ", Opt. Exp., 22, 6478-6485 (2014).

[5] B. Corcoran et al., "Mitigation of Nonlinear Impairments on QPSK Data in Phase-Sensitive Amplified Links", Proc. ECOC'13, We.3.A (2013).

[6] C. Lundström et al., "Fiber optic parametric amplifier With 10-dB net gain without pump dithering", Phot. Tech. Letters, 25, 234-237 (2013).

[7] S. L. I. Olsson et al., "Injection locking-based pump recovery for phase-sensitive amplified links", Opt. Exp., 21, 14512-14529 (2013).

[8] Z. Tong et al., "Towards ultrasensitive optical links enabled by lownoise phase-sensitive amplifiers", Nat. Phot., 5, 430-436 (2011). 